\title{
PROTON TEMPERATURE ANISOTROPY AND MAGNETIC RECONNECTION IN THE SOLAR WIND: EFFECTS OF KINETIC INSTABILITIES ON CURRENT SHEET STABILITY
}

\author{
L. Matteini ${ }^{1,2}$, S. Landi ${ }^{1}$, M. Velli ${ }^{1,3}$, and W. H. Matthaeus ${ }^{4}$ \\ ${ }^{1}$ Dipartimento di Fisica e Astronomia, Università degli Studi di Firenze, Largo E. Fermi 2, I-50125 Florence, Italy \\ ${ }^{2}$ Imperial College London, London SW7 2AZ, UK \\ ${ }^{3}$ Jet Propulsion Laboratory, California Institute of Technology, Pasadena, CA, USA \\ ${ }^{4}$ Bartol Research Institute, Department of Physics and Astronomy, University of Delaware, Newark, DE 19716, USA \\ Received 2012 July 26; accepted 2012 December 6; published 2013 January 17
}

\begin{abstract}
We investigate the role of kinetic instabilities driven by a proton anisotropy on the onset of magnetic reconnection by means of two-dimensional hybrid simulations. The collisionless tearing of a current sheet is studied in the presence of a proton temperature anisotropy in the surrounding plasma. Our results confirm that anisotropic protons within the current sheet region can significantly enhance/stabilize the tearing instability of the current. Moreover, fluctuations associated with linear instabilities excited by large proton temperature anisotropies can significantly influence the stability of the plasma and perturb the current sheets, triggering the tearing instability. We find that such a complex coupling leads to a faster tearing evolution in the $T_{\perp}>T_{\|}$regime when an ion-cyclotron instability is generated by the anisotropic proton distribution functions. On the contrary, in the presence of the opposite anisotropy, fire-hose fluctuations excited by the unstable background protons with $T_{\|}<T_{\perp}$ are not able to efficiently destabilize current sheets, which remain stable for a long time after fire-hose saturation. We discuss possible influences of this novel coupling on the solar wind and heliospheric plasma dynamics.
\end{abstract}

Key words: instabilities - magnetic reconnection - methods: numerical - plasmas - solar wind

Online-only material: color figures

\section{INTRODUCTION}

Particle distribution functions that are not at thermal equilibrium and depart from Maxwellians are frequently observed in space and astrophysical collisionless plasmas. In the weakly collisional solar wind, temperature anisotropies $\left(T_{\perp} \neq T_{\|}\right)$, defined with respect to the local mean magnetic field, are ubiquitous (Marsch et al. 1982). In this framework, microphysics processes generated by nonthermal particle distributions can importantly affect and change the macroscopic evolution of the system (see, for example, Matteini et al. 2012 for a solar wind review). Direct measurements of the ion temperatures in the solar wind (Hellinger et al. 2006; Matteini et al. 2007) and in the magnetosphere (Samsonov et al. 2007) suggest that kinetic instabilities driven by a proton temperature anisotropy play a role in constraining the ratio of parallel and perpendicular temperatures. Such processes act in generating unstable fluctuations that interact with particles and scatter them toward a more isotropic state. Signatures of enhanced wave power associated with kinetic instabilities have been observed in the solar wind (Bale et al. 2009; Wicks et al. 2013), supporting the idea that these mechanisms are active in the plasma expansion. Observations suggest that similar instabilities are also at work for the other solar wind plasma components, as alpha particles (Maruca et al. 2012), and possibly electrons (Štverák et al. 2008), despite that for the latter Coulomb collisions are also expected to significantly play a role (Landi et al. 2012). These kinetic processes are believed to also be relevant in astrophysical plasmas, as galaxy clusters (Schekochihin et al. 2005, 2010; Kunz et al. 2011) and accretion disks (Sharma et al. 2007; Riquelme et al. 2012). Then their investigation in the solar wind, where, thanks to the in situ measurements by spacecraft, we have access to direct comparisons between theory and observations, is also important for the interpretation and modeling of other astrophysical systems.

At the same time, discontinuities of the magnetic field are often observed in the solar wind (e.g., ErdőS \& Balogh 2008).
These current sheet structures are possible sites of magnetic reconnection (e.g., Servidio et al. 2011), and seem to be associated with local plasma heating (Osman et al. 2012b). The role of reconnection in the solar wind is, however, not yet fully understood, and identified reconnection events constitute only a fraction of the total observed discontinuities in the solar wind (e.g., Gosling 2007). At the same time, its contribution to the solar wind heating problem is still an open issue; some authors (Borovsky \& Denton 2011) have found a lack of signatures of preferential plasma heating correlated with strong current sheets. These observations suggest that properties other than exclusively the thickness of the current sheets may play a role in determining the stability of magnetic structures and controlling their dissipation through reconnection processes. A recent observational analysis (Osman et al. 2012a) has shown that the statistical distribution of events associated with current sheets in the solar wind displays some possible correlations with the values of the proton temperature anisotropy, motivating then new modeling and theoretical studies of the coupling between kinetic processes, as the instabilities described above, and solar wind structures. Usually wave-particle interactions and structures are considered independently; however, in nonhomogeneous plasmas particle distribution anisotropies may have an important effect on the stability of gradients associated with current sheets, velocity shears, and density. It is then an open question how small-scale processes develop in nonhomogeneous plasmas and how they interact with coherent structures that are present in the system.

It is known that in collisionless systems current sheets are unstable against tearing instability, a process where the current tends to collapse into filaments. The tearing instability produces magnetic islands that then interact and merge together giving rise to a nonlinear instability phase, where the reconnection process is enhanced. It has recently been pointed out that reconnection processes can significantly influence the local particle distributions; temperature anisotropies associated with 
reconnecting regions are observed in numerical simulations (Aunai et al. 2011; Servidio et al. 2012). Kinetic instabilities generated by these anisotropic distributions have been suggested to be efficient mechanisms able to constrain the size of magnetic islands during reconnection events (Schoeffler et al. 2011) and to play a role in the associated particle acceleration (Drake et al. 2010).

On the other hand, the role of temperature anisotropy and of associated driven instabilities on the onset of magnetic reconnection have been less investigated. Some authors (e.g., Chen \& Davidson 1981; Coppi 1983; Quest et al. 2010) have addressed this problem in the framework of linear theory. In particular, Chen \& Palmadesso (1984) have predicted that in the presence of a $T_{\perp}>T_{\|}$proton anisotropy, the growth rate of the tearing instability is expected to increase with respect to the isotropic case and that the most unstable mode is shifted to larger wavenumbers. On the other side, they also suggested that the tearing instability can be strongly suppressed if the opposite anisotropy $T_{\perp}<T_{\|}$is present in the current sheet. This picture has been confirmed by numerical studies in a hybrid (Ambrosiano et al. 1986) and fluid (Shi et al. 1987) framework.

The aim of this paper is to discuss the stability of small-scale magnetic structures related to current sheets in plasmas that are not at thermal equilibrium, as in the case of the solar wind and heliospheric plasma. Our analysis constitutes a first attempt of coupling in the same numerical model two phenomena, kinetic microinstabilities and magnetic reconnection, which are in general investigated independently. We report results from two-dimensional numerical hybrid simulations that describe the development of a tearing instability of the current in the presence of anisotropic protons, with variable temperature ratio. We find that kinetic instabilities, analogous to those for homogeneous plasma, can develop in the regions away from current sheets and generate waves that subsequently perturb and enhance the observed reconnection process at the current sheets. Following the presentation of the results, we will discuss possible influences of this novel coupling and its nonlinear evolution on the interplanetary and coronal plasma dynamics.

\section{SIMULATION RESULTS}

\subsection{Numerical Setup}

We use a two-dimensional hybrid PIC code (Matthews 1994), describing ions as particles and electrons as a massless charge neutralizing fluid. We initialize the system with a Harris equilibrium model (Harris 1962) for the magnetic field:

$$
\begin{gathered}
B_{y}(x)=B_{0} \tanh (x / l) \\
n(x)=n_{\mathrm{cs}} \operatorname{sech}^{2}(x / l)+n_{b},
\end{gathered}
$$

with a maximum density $n_{0}=1$ at the current sheet. We add a background proton population with $n_{b}=0.2 n_{0}$ uniformly in the box. Units of space and time in the simulations are the ion inertial length $c / \omega_{\mathrm{pi}}\left(\omega_{\mathrm{pi}}\right.$ is the plasma frequency) and the inverse of the proton cyclotron frequency, $\Omega_{\mathrm{cp}}^{-1}$, respectively. The adopted simulation box is $50 \mathrm{c} / \omega_{\mathrm{pi}}$ long in the $x$-direction and $200 c / \omega_{\text {pi }}$ in the $y$-direction, with a $100 \times 200$ grid and spatial resolution $\Delta x=0.5 c / \omega_{\mathrm{pi}}$ and $\Delta y=1 c / \omega_{\mathrm{pi}}$; we use $10^{3}$ particles per cell. The box has periodic boundaries in both directions and thus contains two current sheets of width $l=1.5 c / \omega_{\mathrm{pi}}$. Note that the spatial separation of the current sheets is such that they do not influence each other during the development of the tearing instability or of the anisotropy-driven
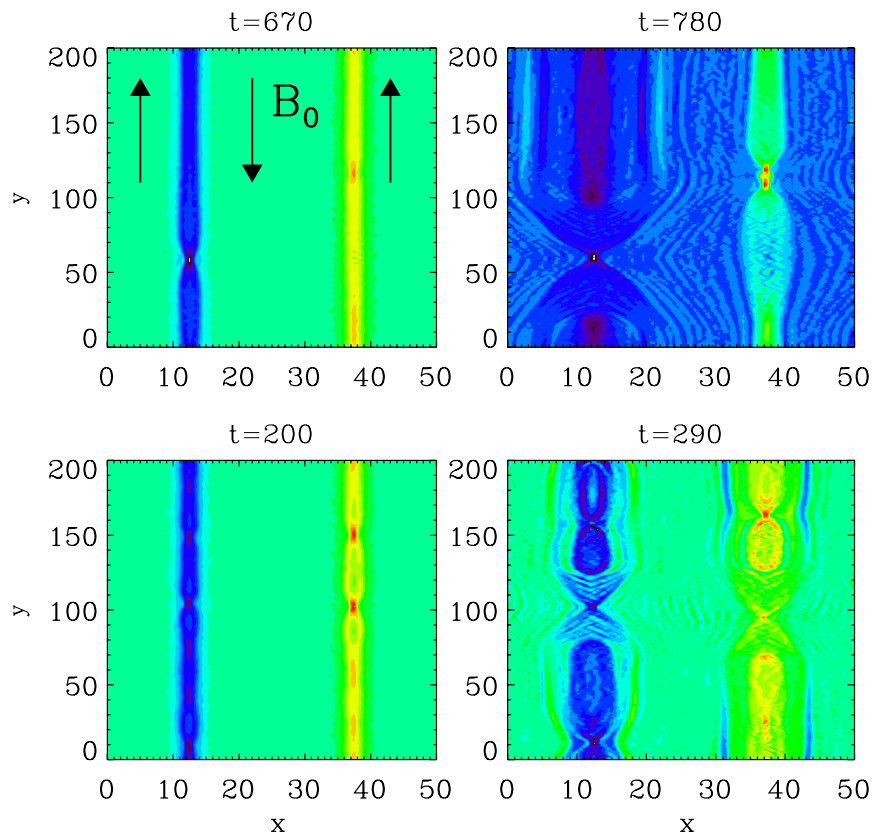

Figure 1. Evolution of tearing mode in cases with isotropic proton temperature (top panels) and anisotropic protons in the current sheet region (bottom panels), out of the plane current $J_{z}$ during the linear (left) and nonlinear (right) phases of the instability. The background plasma is isotropic. Arrows in the top left panel indicate the local direction of the ambient magnetic field $B_{y}(x)=B_{0}$ in the homogeneous regions around the current sheets.

(A color version of this figure is available in the online journal.)

kinetic instabilities. As expected, interactions between islands associated with distinct sheets are later observed, but only at the final stage of the nonlinear phase of the runs.

The parallel beta $\beta_{\|}=8 \pi n k_{B} T_{\|} / B_{0}^{2}$ of the background plasma is set equal to 1 and the electron beta to 0.5 . The temperature anisotropy $\left(T_{\perp} / T_{\|}\right)$, with respect to the equilibrium magnetic field, of both populations is varied in order to test the stability of the magnetic configuration with respect to different plasma conditions. Note that in such a configuration there are regions between the current sheets of homogeneous and constant $B_{0}$ magnetic field, aligned with $\pm y$ (see top left panel of Figure 1), where fluctuations generated by small-scale processes can develop and propagate.

\subsection{Simulations with Isotropic Background}

We start our analysis with a simulation in which all the plasma is isotropic and focus on the development of a tearing instability from the initial configuration. In the top panels of Figure 1, we report the evolution of the current sheets at two simulation times. At the top left, at time $t=670$, at the end of the linear growth a tearing mode with $m=1$ is observed; at a later time, $t=780$, the dynamics becomes nonlinear and a consequent strong deformation of the current is visible in the top right panel. We underline that the instability passes through a long linear phase and highly nonlinear only after $t \sim 650$. This assures that, although the adopted initial conditions are not an exact kinetic equilibrium (e.g., Daughton 1999; Belmont et al. 2012), we do not observe other processes destabilizing the system on a shorter timescale than the classic tearing instability.

We consider now the case of a plasma with a temperature anisotropy. We have performed simulations using several different initial temperature anisotropies for the current sheet population. At this stage the background proton population is 

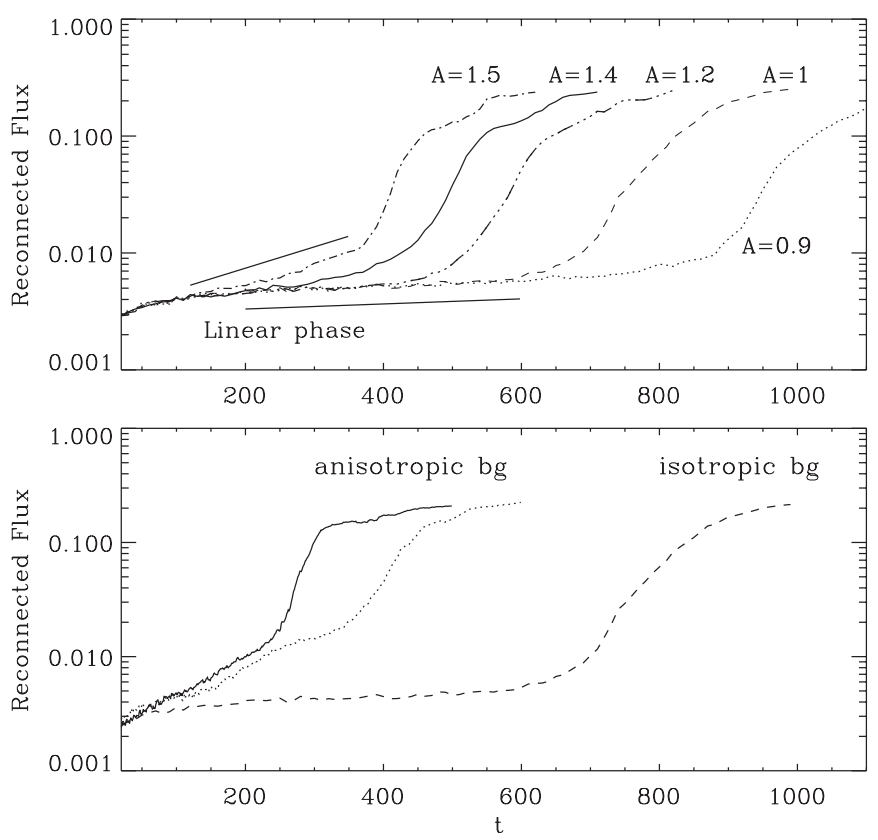

Figure 2. Top panel: temporal evolution of the reconnected flux for runs with isotropic background and different initial current sheet anisotropy $A=$ $T_{\perp} / T_{\|}$. Lines encode runs with $T_{\perp}=0.9 T_{\|}$(dotted), $T_{\|}=T_{\perp}$ (dashed), $T_{\perp}=1.2 T_{\|}$(dash-dot-dot-dotted), $T_{\perp}=1.4 T_{\|}$(solid), and $T_{\perp}=1.5 T_{\|}$(dashdotted). Bottom panel: reconnected flux in the presence of unstable anisotropic background protons with $T_{\perp}=2 T_{\|}$, for a thin $(l=1.5$, solid) and a thicker $(l=3$, dotted) current sheet; the full isotropic plasma case is reported in dashed line as a reference.

maintained isotropic. Our investigation qualitatively confirms the picture of Chen \& Palmadesso (1984), with a faster tearing for larger perpendicular temperature, and a weaker instability if the parallel temperature is larger. Increasing the anisotropy with larger perpendicular temperatures leads to a shift of the most unstable tearing mode to larger wavenumbers with an increase of the instability growth rate. This is illustrated in the bottom panels of Figure 1 that reports the evolution of $J_{z}$ for a simulation with $T_{\perp}=2 T_{\|}$at times $t=200$ (left) and $t=290$ (right). Starting with such an initial anisotropy for the proton population at the current sheet gives rise to the excitation of a higher mode with $m=6$, and this occurs on a significantly faster timescale compared to the isotropic case (top panels of the figure), leading to the formation of several magnetic islands along the current sheets; subsequently, these are observed to merge giving rise to a strong nonlinear phase (right panel) already at $t \sim 290$.

The top panel of Figure 2 summarizes the results obtained for various initial temperature anisotropies, reporting the reconnected flux evolution for different runs. The dotted line refers to the isotropic reference case. From right to left, the lines encode $T_{\perp}=0.9 T_{\|}$(dotted), $T_{\|}=T_{\perp}$ (dashed), $T_{\perp}=1.2 T_{\|}$(dashdot-dot-dotted), $T_{\perp}=1.4 T_{\|}$(solid), and $T_{\perp}=1.5 T_{\|}$(dashdotted). Note that in the figure the linear phase of the instability ("tearing") corresponds to the flatter initial part, and that the strong increase of the flux identifies the nonlinear phase, with large amplitude island growth and merging. We observe that when increasing the anisotropy (with $T_{\perp} / T_{\|}>1$ ), the linear slope steepens and the nonlinear stage is significantly accelerated. On the other side, when $T_{\|}>T_{\perp}$, the whole process is delayed. In agreement with previous studies (Chen \& Palmadesso 1984; Ambrosiano et al. 1986), our results confirm that the linear growth rates also depend on the current sheet thickness; we have checked that all the growth rates reported in the figure significantly decrease when taking larger sheet widths.

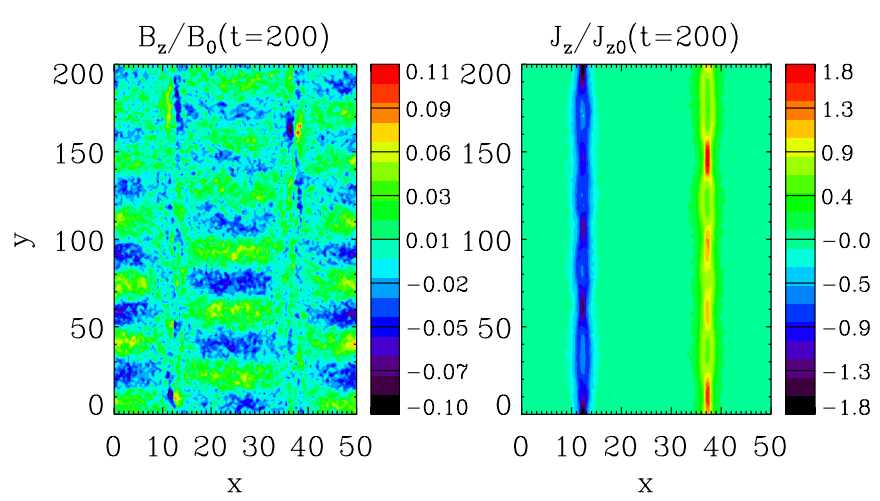

Figure 3. Right panel: $B_{z}$ component of ion-cyclotron fluctuations generated by unstable background protons with $T_{\perp}=2 T_{\|}$, at $t=200$. Left panel: modulation of the current $J_{z}$ at the same time.

(A color version of this figure is available in the online journal.)

\subsection{Simulations with Anisotropic Background}

It is worth noting that when extending the investigation to large temperature ratios the plasma can become unstable with respect to kinetic instabilities driven by enhanced thermal anisotropies. In particular, an ion-cyclotron instability can develop in a $T_{\perp}>T_{\|}$condition (e.g., Gary et al. 2003). This mechanism produces fluctuations that scatter particles and reduce the initial anisotropy bringing the system toward a marginal stable condition. (Note that a mirror instability can be also excited in the $T_{\perp}>T_{\|}$regime (e.g., Hellinger 2007).) It is then interesting to investigate what happens in the adopted configuration when the injected anisotropy is such to destabilize this linear instability and what are its consequences on the stability of the current sheets. To deal with this aspect, we have performed simulations where the temperature anisotropy is not confined in the current sheet region, as in previous section, but it is extended to the whole background plasma. Note that when this new condition is studied in a regime of stability for the background protons (i.e., weak anisotropy), no peculiar differences are observed in the evolution of the tearing instability with respect to the previous cases; the surrounding plasma remains quiet and homogeneous, because stable, while the current is still destabilized with rates consistent with those reported in top panel of Figure 2, which correspond to an isotropic background plasma.

On the contrary, when the initial anisotropy of the background is such that the uniform regions of plasma are unstable (with $T_{\perp}=2 T_{\|}$), we observe in the early stages of the simulation the generation of an ion-cyclotron mode, corresponding to the most unstable wavenumber, that starts to propagate along the local magnetic field. The left panel of Figure 3 reports the outof-the-plane $B_{z}$ fluctuations associated with the ion-cyclotron instability which characterizes the region of homogeneous magnetic field $B_{y}$ bounded by the current sheets. As soon as these are generated, such fluctuations start to perturb the current sheet profiles (right panel); as we will discuss later, thanks to the symmetry imposed by the ion-cyclotron fluctuations at the two sides of the sheet, the forced perturbation consists of a tearing modulation of the current and since this occurs at a scale close to the most unstable current tearing wavenumber, the instability is efficiently triggered. As a consequence, this coupling leads to a fast driving of the tearing instability, as shown by the solid line in the bottom panel of Figure 2, which reports the time evolution of the reconnected flux; the dissipation of the current occurs on a shorter time than in the case when the background 

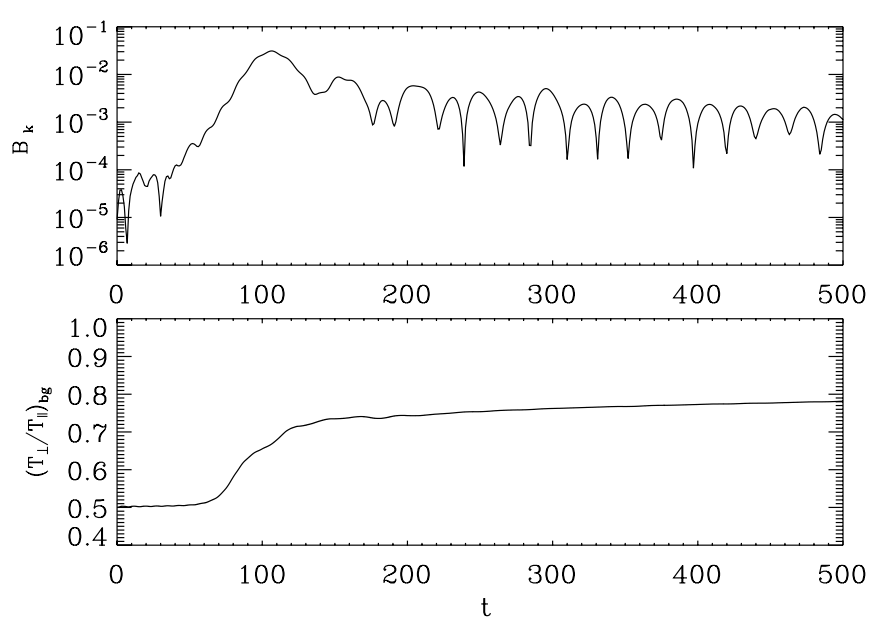

Figure 4. Run with initial background anisotropy $T_{\|}=2 T_{\perp}$ and $\beta_{\|}=4$, predicted unstable for fire-hose instability. Top panel: history of the dominant component of the Fourier transform of $B_{z}$, associated with a fire-hose unstable mode. Bottom panel: evolution of the temperature anisotropy of background protons.

is isotropic (dashed line). Moreover, it is remarkable that in the linear part, the growth of the tearing is driven by the rms of the $B_{z}$ fluctuations excited by the ion-cyclotron instability. This result demonstrates that unstable fluctuations generated by ion-cyclotron instability importantly influence the stability of the magnetic equilibrium and they can directly accelerate the development of the tearing instability.

It is also important to underline that, while the unforced linear tearing depends strongly on the thickness of the current, on the other side the excitation of fluctuations by kinetic instabilities depends only on the plasma conditions outside the current sheet (temperature anisotropy and beta). As a consequence, the trigger of the tearing instability by ion-cyclotron fluctuations results approximatively independent of the sheet thickness, as shown by the dotted line in the bottom panel of Figure 2, reporting the reconnected flux for a case with a larger thickness $l=3$ but same proton anisotropy in the background. Despite the increase of sheet thickness and unlike the classical linear tearing, we still recover a fast tearing, with a growth compatible to that of the thinner sheet case. Note that the only difference consists of a longer linear phase, since the tearing of the thicker current needs a longer time to become nonlinear.

An important question at this point concerns whether the presence of any fluctuations induced by a small-scale process would be sufficient to trigger such a faster destabilization of the current (Coppi 1983), and if this may occur independently of the sign of the plasma anisotropy. To address this issue, we have considered a case with the opposite temperature anisotropy, where, due to a large $T_{\|}>T_{\perp}$ anisotropy, protons of the background plasma are predicted unstable with respect to a firehose instability by the linear Vlasov theory. In this situation $\left(T_{\|}=2 T_{\perp}\right.$ and $\left.\beta_{\|}=4\right)$, a strong fire-hose mode is soon generated in the box: the top panel of Figure 4 reports the time history of the $B_{z}$ Fourier component corresponding to this mode, showing its exponential growth in time for $t<100$. The growth rate associated with this mode is in qualitative agreement with the linear Vlasov prediction for homogeneous plasma, even if a deviation is observed, probably due to the more complicated plasma configuration adopted in the simulation.

As expected, the effect of this instability is to scatter particles and reduce their temperature anisotropy, driving the plasma to a more isotropic marginal stable state (lower panel). The
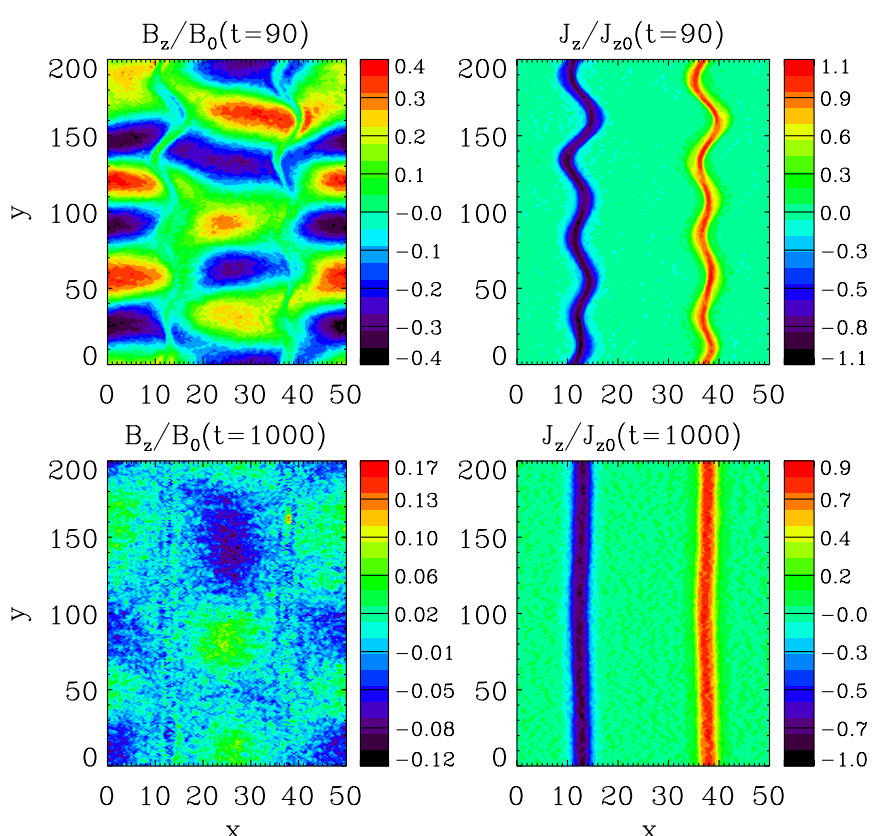

Figure 5. Evolution of $B_{z}$ (left) and $J_{z}$ (right) for a simulation with anisotropic protons in the background with $T_{\|}=2 T_{\perp}$ (same run as in Figure 4), during the linear phase of the fire-hose instability (top panels) and at a late post-saturation time (bottom)

(A color version of this figure is available in the online journal.)

saturation of the magnetic field fluctuations at $t \gtrsim 100$ corresponds to the stabilization of the plasma; after this stage, the proton anisotropy remains constant and the fire-hose fluctuations are slowly damped in time. Note that the observed behavior, which is in good agreement with the standard evolution of this instability found in simulations of homogeneous plasma (e.g., Matteini et al. 2006), is recovered here in the case of a non-homogeneous plasma, supporting the idea that such processes can be at work in real plasmas, like the solar wind, that are characterized by nonthermal distributions and small-scale coherent structures.

The activity of the fire-hose instability described in the simulation influences and perturbs the magnetic field structure; it is worth noting that the fire hose is an instability of magnetic field oscillations (e.g., Davidson \& Völk 1968) and thus leads to an oscillatory modulation of magnetic field lines. As a consequence, its influence on the current sheets is to produce a kink deformation of $J_{z}$, which becomes more and more significant as the fire-hose unstable mode grows and reaches a macroscopic scale before saturation, as shown in the top panels of Figure 5. It is remarkable that this macroscopic perturbation of the current does not give rise to any tearing instability of the current sheets. On the contrary, these are observed to oscillate passively under the effect of the fire-hose activity. Such kinktype oscillations are later damped as soon as, according to Figure 4, the fire-hose mode is reabsorbed by the plasma at longer times. As shown in the bottom panels of Figure 5, at $t=1000$, after the oscillatory stage, the system appears stable again and the current sheets recover their initial equilibrium configuration as at the beginning of the simulation. Starting from this late condition, a tearing instability can then eventually develop and produce the collapse of the current, but only in a significantly longer time than in the cases that are stable against the fire hose.

To investigate in more detail the origin of the different modulation of the current sheets observed in the simulations, 
the color contour in Figure 5 reports the magnetic fluctuations $B_{x}$ associated with ion-cyclotron (left) and fire-hose (right) instabilities in the background plasma. The superimposed black solid line contours encode the density of protons within the current sheet. Arrows identify the local direction of the electric field generated by the kinetic instability fluctuations. As shown by the figure, the modulation of the current sheet is very well correlated to these fluctuations, suggesting that the resulting deformation of the current is guided by the background activity, leading to a different evolution according to the different symmetry of the fluctuations. The spatial structure of the $x$-component of the magnetic field, $B_{x}$, is a relevant quantity in our analysis since, through the term $J \times B=c / 4 \pi(\nabla \times B) \times B$, it generates an electric field component pushing particle toward and away from the current sheet. In our case, this component of $E_{x}$ is proportional to $\left(\partial_{y} B_{x}\right) B_{0}$, which clearly depends on the symmetry of $B_{x}$ across the discontinuity. When $B_{x}$ is symmetric through the current sheet (left panel, ion-cyclotron instability case), then the associated electric field has opposite sign on the two sides (due to the orientation change in $B_{0}$ ), leading to diverging or converging flows. On the contrary, when $B_{x}$ is antisymmetric (right panel, fire-hose case), the electric field does not change orientation across the discontinuity (both $B_{x}$ and $B_{0}$ change sign) and gives rise to an oscillation along $y$. The local direction of this contribution to the electric field is reported by the arrows in the figure and well explains either the kink or the tearing deformation of the current under the effects of the external, anisotropy driven, unstable fluctuations.

\section{SUMMARY AND CONCLUSIONS}

In summary, we have investigated the properties of current sheets embedded in an anisotropic plasma, focusing on the coupling between small-scale processes, temperature anisotropy driven kinetic instabilities, and evolution of plasma structures leading to magnetic reconnection. We have tested the stability of an initial Harris equilibrium as a function of the temperature ratio that characterizes protons in the current sheet region and in the background plasma. In agreement with previous works (Chen \& Palmadesso 1984), we find that the temperature anisotropy plays an important role in determining the stability of the current with respect to the tearing instability. A $T_{\perp}>T_{\|}$ condition within the current sheet leads to faster tearing, and at larger wavenumbers, while the opposite $T_{\perp}<T_{\|}$condition has a stabilizing effect. Moreover, the development of kinetic instabilities that are driven when these temperature anisotropies are distributed in the background plasma changes further the properties of the system.

It is interesting to note that although the adopted simulation domain is not homogeneous, as is usually done for kinetic instabilities studies (e.g., Matteini et al. 2006), they are observed to develop anyway; this is because in the box there are regions where the magnetic field is locally uniform (see, for example, Figure 1) and even if restricted to narrow channels (few inertial lengths), unstable fluctuations can be excited. This suggests that the development of those instabilities, as predicted for a homogeneous Vlasov plasma, is robust and may characterize also the evolution of realistic, non-homogeneous systems as space and astrophysical plasmas.

We have shown that ion-cyclotron fluctuations, generated in the presence of an initial unstable $T_{\perp}>T_{\|}$anisotropy in the background, are able to trigger the destabilization of the current and drive a tearing instability (Figure 3) that emerges more rapidly than it does in the case in which the anisotropy is
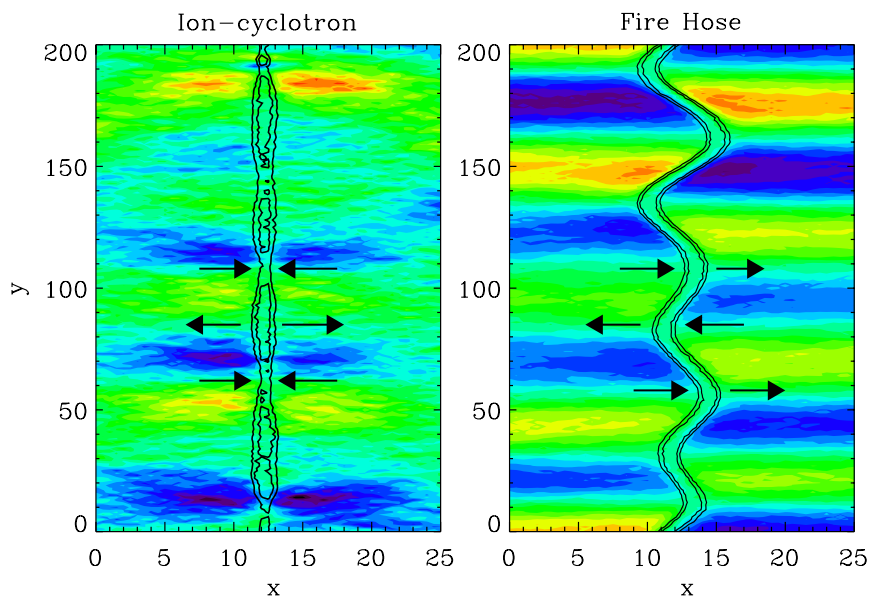

Figure 6. Color contours encode the background $B_{x}$ fluctuations generated by ion-cyclotron (left) and fire-hose (right) instabilities. The density of protons at the current sheet at the same simulation time is reported on the superimposed black solid line contour. The arrows show the local direction of the resulting contribution to the electric field component $E_{x}$ generated by the magnetic fluctuations in the figure.

(A color version of this figure is available in the online journal.)

confined to the current sheet regions and ion-cyclotron modes are not excited. Moreover, since such a trigger only depends on the conditions of the background plasma, the tearing instability forced by those fluctuations results to be roughly independent of the current sheet thickness. On the other hand, we have also shown that fire-hose fluctuations that are generated when an excessive $T_{\perp}<T_{\|}$anisotropy characterizes the plasma are not able to trigger enhanced instability of the current. In that case, due to the nature of those modes (Figure 6), the observed deformation is of the kink-type and does not produce favorable conditions for tearing. As a consequence, the current sheets remain stable even when such fluctuations are later dissipated (Figure 5), and, remarkably, for a longer time than the isotropic or fire-hose stable case.

We propose that this novel coupling, leading to different evolution according to value of the temperature anisotropy, may take place in the solar wind, where alternatively conditions of $T_{\perp}>T_{\|}$and $T_{\perp}<T_{\|}$for protons are observed (Marsch et al. 1982). Kinetic instabilities, like fire hose and ion-cyclotron, are believed to play a role in regulating such proton temperature anisotropy along the solar wind expansion (Hellinger et al. 2006; Matteini et al. 2007; Bale et al. 2009) and fluctuations excited by these instabilities can then importantly influence the properties of the plasma. Data analysis of small-scale fluctuations and particle anisotropies in the solar wind should be complemented with local wind structure information. This can be relevant in the study of reconnection exhausts as a function of magnetic shear (e.g., Gosling et al. 2006). We suggest, for example, that small-scale structures, discontinuities, and current sheets may be more observable, because stable, when the plasma is in a $T_{\perp}<T_{\|}$regime, as we have shown that in this regime the plasma is more stable and even external large amplitude fluctuations, like fire hose, are not able to drive the current collapse, in possible agreement with the recent solar wind observations of Osman et al. (2012a). On the ecliptic, this can be also relevant for the dynamics of heliospheric current sheet that is mainly surrounded by slow wind plasma, displaying a majority of $T_{\|}>T_{\perp}$ (Hellinger et al. 2006). On the contrary, we expect fast streams observed at high latitude and characterized by protons with highly anisotropic cores with $T_{\perp}>T_{\|}$(Marsch et al. 2004; 
Hellinger et al. 2011) constitute a less favorable condition for stable small-scale magnetic structure as current sheets, as well as planetary magnetosheath where enhanced $T_{\perp}>T_{\|}$anisotropies are often observed for the downstream protons (Samsonov et al. 2007).

The coupling between particle properties and the evolution of small-scale magnetic structures, as described in this work, will be more deeply investigated with the help of measurements from the future heliospheric explorations, such as Solar Orbiter and Solar Probe Plus, where combined particle and field highresolution observations are planned.

The research leading to these results has received funding from the European Commission's Seventh Framework Programme (FP7/2007-2013) under the grant agreement SHOCK (project number 284515), from the US NSF SHINE program, and from the Science and Technology Facilities Council (STFC). It was also carried out in part at the Jet Propulsion Laboratory, California Institute of Technology, under a contract with the National Aeronautics and Space Administration.

\section{REFERENCES}

Ambrosiano, J., Lee, L. C., \& Fu, Z. F. 1986, JGR, 91, 113 Aunai, N., Belmont, G., \& Smets, R. 2011, JGR, 116, 9232 Bale, S. D., Kasper, J. C., Howes, G. G., et al. 2009, PhRvL, 103, 211101 Belmont, G., Aunai, N., \& Smets, R. 2012, PhPl, 19, 022108 Borovsky, J. E., \& Denton, M. H. 2011, ApJL, 739, 61

Chen, J., \& Davidson, R. C. 1981, PhFl, 24, 2208

Chen, J., \& Palmadesso, P. 1984, PhFl, 27, 1198

Coppi, B. 1983, ApJL, 273, 101

Daughton, W. 1999, PhPl, 6, 1329

Davidson, R. C., \& Völk, H. J. 1968, PhFl, 11, 2259

Drake, J. F., Opher, M., Swisdak, M., \& Chamoun, J. N. 2010, ApJ, 709, 963

ErdőS, G., \& Balogh, A. 2008, AdSpR, 41, 287

Gary, S. P., Yin, L., Winske, D., et al. 2003, JGR, 108, 1068

Gosling, J. T. 2007, ApJL, 671, 73
Gosling, J. T., Eriksson, S., Skoug, R. M., McComas, D. J., \& Forsyth, R. J. 2006, ApJ, 644, 613

Harris, E. G. 1962, NCimB, 23, 115

Hellinger, P. 2007, PhPl, 14, 2105

Hellinger, P., Matteini, L., Štverák, Š., Trávníček, P. M., \& Marsch, E. 2011, JGR, 116, 9105

Hellinger, P., Trávníček, P., Kasper, J. C., \& Lazarus, A. J. 2006, GeoRL, 33, 9101

Kunz, M. W., Schekochihin, A. A., Cowley, S. C., Binney, J. J., \& Sanders, J. S. 2011, MNRAS, 410, 2446

Landi, S., Matteini, L., \& Pantellini, F. 2012, ApJ, 760, 143

Marsch, E., Ao, X.-Z., \& Tu, C.-Y. 2004, JGR, 109, 4102

Marsch, E., Schwenn, R., Rosenbauer, H., et al. 1982, JGR, 87, 52

Maruca, B. A., Kasper, J. C., \& Gary, S. P. 2012, ApJ, 748, 137

Matteini, L., Hellinger, P., Landi, S., Trávnícek, P., \& Velli, M. 2012, SSRv, 172,373

Matteini, L., Landi, S., Hellinger, P., \& Velli, M. 2006, JGR, 111, 10101

Matteini, L., Landi, S., Hellinger, P., et al. 2007, GeoRL, 34, 20105

Matthews, A. P. 1994, JCoPh, 112, 102

Osman, K. T., Matthaeus, W. H., Hnat, B., \& Chapman, S. C. 2012a, PhRvL, 108,261103

Osman, K. T., Matthaeus, W. H., Wan, M., \& Rappazzo, A. F. 2012b, PhRvL, 108,261102

Quest, K. B., Karimabadi, H., \& Daughton, W. 2010, PhPl, 17, 022107

Riquelme, M. A., Quataert, E., Sharma, P., \& Spitkovsky, A. 2012, ApJ, 755, 50

Samsonov, A. A., Alexandrova, O., Lacombe, C., Maksimovic, M., \& Gary, S. P. 2007, AnGeo, 25, 1157

Schekochihin, A. A., Cowley, S. C., Kulsrud, R. M., Hammett, G. W., \& Sharma, P. 2005, ApJ, 629, 139

Schekochihin, A. A., Cowley, S. C., Rincon, F., \& Rosin, M. S. 2010, MNRAS, 405, 291

Schoeffler, K. M., Drake, J. F., \& Swisdak, M. 2011, ApJ, 743, 70

Servidio, S., Greco, A., Matthaeus, W. H., Osman, K. T., \& Dmitruk, P. 2011, JGR, 116, 9102

Servidio, S., Valentini, F., Califano, F., \& Veltri, P. 2012, PhRvL, 108, 045001

Sharma, P., Quataert, E., Hammett, G. W., \& Stone, J. M. 2007, ApJ, 667, 714

Shi, Y., Lee, L. C., \& Fu, Z. F. 1987, JGR, 92, 12171

Štverák, Š., Trávníček, P., Maksimovic, M., et al. 2008, JGR, 113, 3103

Wicks, R. T., Matteini, L., Horbury, T. S., Hellinger, P., \& Roberts, A. D. 2013, Proc. Thirteenth International Solar Wind Conference (Melville, NY: AIP), in press 